06

\title{
Влияние дозы имплантации ионов азота на концентрацию точечных дефектов, введенных в слои GaAs
}

\author{
() Н.А. Соболев, Б.Я. Бер, Д.Ю. Казанцев, А.Е. Калядин, \\ К.В. Карабешкин, В.М. Микушкин, В.И. Сахаров, \\ И.Т. Серенков, Е.И. Шек, Е.В. Шерстнев, Н.М. Шмидт
}

Физико-технический институт им. А.Ф. Иоффе РАН, Санкт-Петербург,

Россия

E-mail: nick@sobolev.ioffe.rssi.ru

Поступило в Редакцию 29 ноября 2017 г.

С помощью методов масс-спектрометрии вторичных ионов и резерфордовского обратного рассеяния протонов измерены профили концентрации атомов азота и исследована дефектная структура эпитаксиальных слоев GaAs, имплантированных ионами $\mathrm{N}^{+}$с энергией $250 \mathrm{keV}$ и дозами $5 \cdot 10^{14}-5 \cdot 10^{16} \mathrm{~cm}^{-2}$. Установлено, что аморфизации имплантируемых слоев при дозах выше расчетного порога аморфизации не происходит, концентрация образовавшихся точечных дефектов значительно меньше расчетной, а характерной особенностью концентрационных профилей дефектов является их высокая концентрация в приповерхностном слое.

DOI: $10.21883 /$ PJTF.2018.13.46326.17139

Разбавленные твердые растворы $\mathrm{GaAs}_{1-x} \mathrm{~N}_{x}$ рассматриваются в качестве перспективных кандидатов для создания эффективных источников излучения в зеленой области спектра и приборов для инфракрасной оптоэлектроники [1]. Используются различные методы получения этих растворов, в том числе молекулярно-пучковая эпитаксия [2,3], металлоорганическое химическое напыление [4] и ионная имплантация [1,5-9]. В настоящее время сведения о получении конкурентоспособных приборных структур на основе данных твердых растворов практически отсутствуют. Весьма ограничена информация о дефектах и их влиянии на свойства таких твердых растворов. Ионная имплантация является простым и эффективным методом введения азота в GaAs с концентрацией выше равновесной, но при этом важную роль в решении задачи 
получения твердых растворов приборного качества играют контроль вводимых имплантационных дефектов и методы их устранения. Однако в публикациях практически отсутствует информация о введенных имплантационных дефектах. В настоящей работе приведены первые результаты исследования дефектов в слоях GaAs: N, полученных при имплантации ионов азота с разными дозами.

Имплантация ионов $\mathrm{N}^{+}$проводилась при комнатной температуре с энергией $250 \mathrm{keV}$, плотностью тока $\sim 0.45 \mu \mathrm{A} / \mathrm{cm}^{2}$ и дозами $5 \cdot 10^{14}$, $5 \cdot 10^{15}$ и $5 \cdot 10^{16} \mathrm{~cm}^{-2}$ на ускорителе фирмы High Voltage Engineering Europe в слои GaAs толщиной $\sim 40 \mu \mathrm{m}$, выращенные методом газофазной эпитаксии в хлоридной системе на сильнолегированных (100) GaAs-подложках. Проецированный пробег ионов азота $R_{p}$, согласно программе TRIM [10], составляет $417 \mathrm{~nm}$. Пучок ионов направлялся под углом $7^{\circ}$ от нормали к имплантируемой поверхности, чтобы избежать эффекта каналирования. Концентрационные профили атомов азота измерялись с помощью метода вторичной ионной масс-спектроскопии (SIMS) на установке Cameca IMS 4f. Дефектная структура имплантированных слоев исследовалась с помощью метода резерфордовского обратного рассеяния (RBS) протонов с энергией $227 \mathrm{keV}$. Кремниевый детектор с энергетическим разрешением $\sim 3.5 \mathrm{keV}$ регистрировал протоны, рассеянные на угол $170^{\circ}$. Измерение энергетических спектров обратнорассеянных протонов проводилось в режимах каналирования и псевдослучайного направления пучка (далее каналирующий и случайный режимы). Длительность измерения RBS-спектров каждого из образцов изменялась в диапазоне $0.5-3 \mathrm{~h}$. Было установлено, что результаты измерений не зависели от длительности, т. е. влияние диагностического пучка на структуру исследуемого объекта отсутствовало. Расчет профилей концентрации введенных точечных дефектов проводился с помощью процедуры, предложенной в [11].

На рис. 1 представлен измеренный с помощью метода SIMS профиль атомов азота в образце $\mathrm{GaAs}$, имплантированном с дозой $5 \cdot 10^{14} \mathrm{~cm}^{-2}$. Распределение ионов азота, рассчитанное с помощью программы TRIM, также приведено на рисунке. Видно, что экспериментальный и расчетный профили, а также их основные параметры (положение максимума, максимальная концентрация атомов азота и полуширина распределения) довольно хорошо (в пределах $\pm 10 \%$ ) совпадают. Аналогичное совпадение экспериментального и расчетного профилей наблюдалось и для образца со средней дозой имплантации.

Письма в ЖТФ, 2018, том 44, вып. 13 


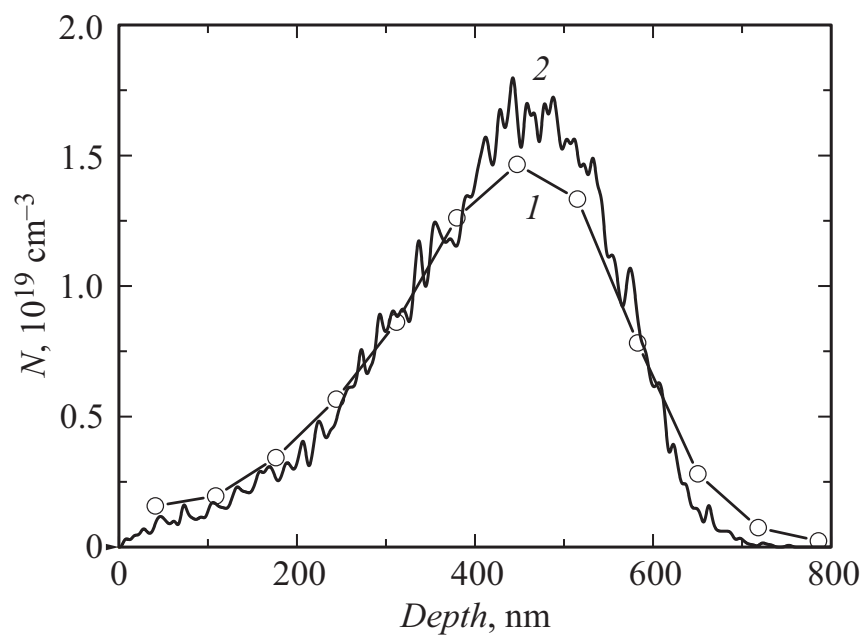

Рис. 1. Измеренные методом SIMS (1) и рассчитанные с помощью программы TRIM (2) концентрационные профили атомов азота, имплантированных в $\mathrm{GaAs}$ с энергией $250 \mathrm{keV}$ и дозой $5 \cdot 10^{14} \mathrm{~cm}^{-2}$.

Для образца с наибольшей дозой имплантации экспериментальный профиль и расчетный TRIM-профиль при концентрациях атомов азота выше $1 \cdot 10^{20} \mathrm{~cm}^{-3}$ не совпадают. Это может быть связано с тем обстоятельством, что при таких больших дозах имплантируемых ионов происходит существенное изменение структуры слоя, нарушающее условия применимости методики стандартного количественного анализа в SIMS.

Спектры обратного рассеяния протонов в образцах после имплантации ионов азота, измеренные в случайном и каналирующем режимах, приведены на рис. 2 (кривые 1-4). Анализ RBS-спектров образцов GaAs: N показал, что аморфизации имплантируемых слоев не происходит (амплитуда случайного спектра существенно превосходит амплитуды каналированных спектров). Каналированные спектры образцов, имплантированных с дозами $5 \cdot 10^{14}$ и $5 \cdot 10^{15} \mathrm{~cm}^{-2}$, практически совпадают. Профили концентрации точечных дефектов, рассчитанные из RBS-спектров и нормированные на число атомов в $1 \mathrm{~cm}^{3}$, для образцов с дозами имплантации $5 \cdot 10^{14}$ и $5 \cdot 10^{15} \mathrm{~cm}^{-2}$ приведены на рис. 3

Письма в ЖТФ, 2018, том 44, вып. 13 


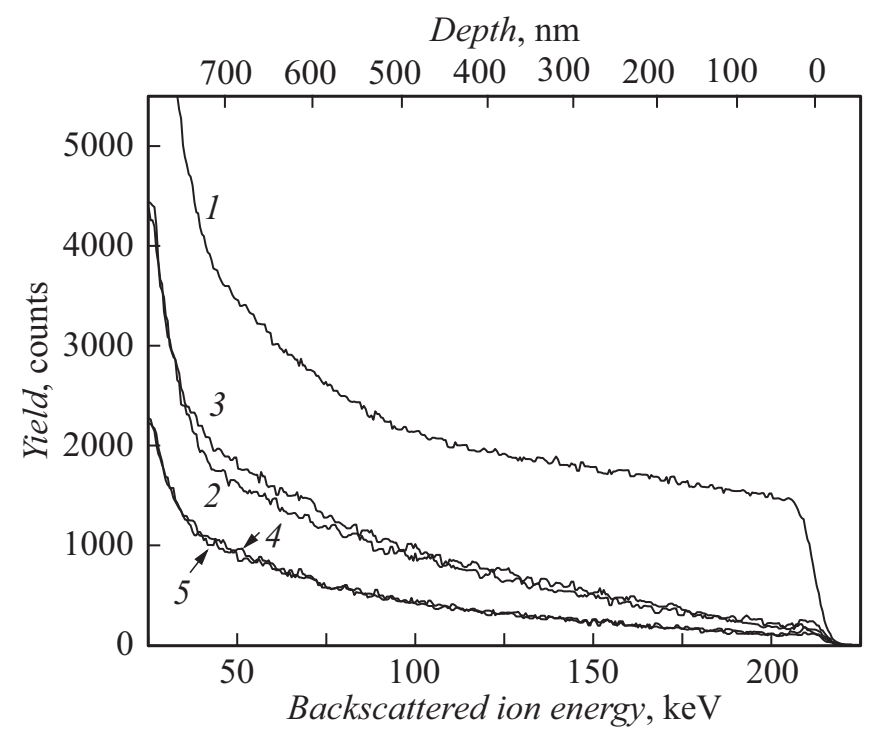

Рис. 2. Энергетические RBS-спектры: случайный (1) и каналированные вдоль оси $\langle 100\rangle$ после имплантации ионов азота с дозами $5 \cdot 10^{14}(2), 5 \cdot 10^{15}(3)$ и $5 \cdot 10^{16} \mathrm{~cm}^{-2}(4)$. Для сравнения показан каналированный спектр образца перед имплантацией $(5)$.

(кривые 1 и 2 соответственно). Был получен необычный результат, поскольку увеличение дозы имплантации на порядок не приводит к изменению концентрационных профилей. Кроме того, на глубинах меньше $200 \mathrm{~nm}$ наблюдается существенное увеличение концентрации дефектов по направлению к поверхности. Такое возрастание концентрации дефектов в приповерхностном слое в процессе имплантации свидетельствует о диффузии атомов из объема к поверхности. При исследовании имплантированных образцов на микроскопе с большим $(\times 1000)$ увеличением и на сканирующем электронном микроскопе обнаружены выделения, которые, по-видимому, связаны с диффузией атомов $\mathrm{Ga}$. Ранее аналогичный эффект диффузии $\mathrm{Ga}$ и его преципитации на поверхности наблюдался нами при имплантации ионов $\mathrm{Er}$ в сверхрешетки $\mathrm{AlGaN} / \mathrm{GaN}$ [12]. Такие эффекты проявлялись и при имплантации различных ионов в другие Ga-содержащие соединения $\mathrm{A}^{\mathrm{III}} \mathrm{B}^{\mathrm{V}}$ [13].

Письма в ЖТФ, 2018, том 44, вып. 13 


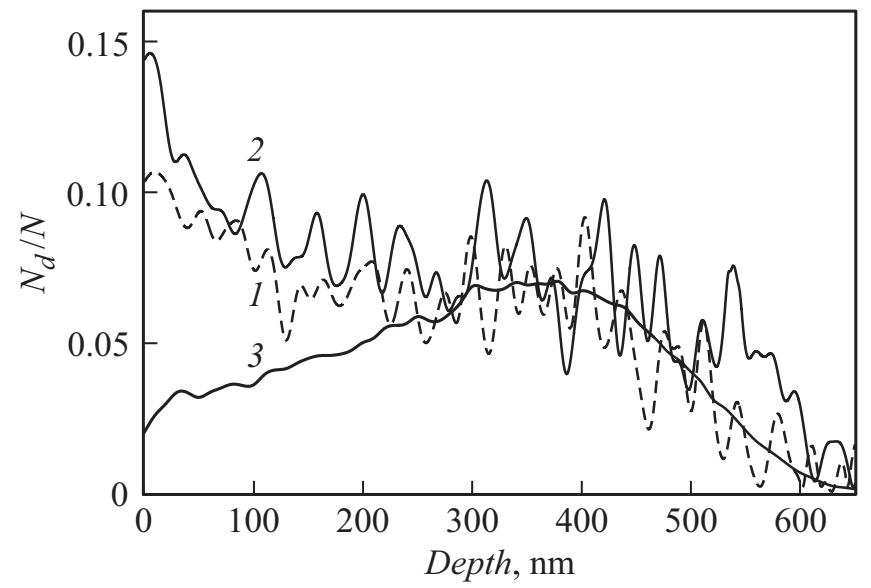

Рис. 3. Концентрационные профили дефектов, введенных имплантацией в структуры GaAs: N при дозах $5 \cdot 10^{14}(1)$ и $5 \cdot 10^{15} \mathrm{~cm}^{-2}(2)$, и расчетная кривая для дозы имплантации $1 \cdot 10^{14} \mathrm{~cm}^{-2}(3)$.

Кроме того, взаимодействие присутствовавшего до имплантации в микротрубках эпитаксиальных слоев GaAs избыточного галлия с азотом может приводить к избыточным напряжениям в этих областях и, как следствие, к увеличению концентрации дефектов. На рис. 3 также приведен рассчитанный с помощью программы TRIM профиль дефектов после имплантации ионов азота с дозой $1 \cdot 10^{14} \mathrm{~cm}^{-2}$ (кривая 3). Практически полное совпадение экспериментальных и расчетного профилей на глубинах более $250 \mathrm{~nm}$ позволяет предположить, что при дозах выше $1 \cdot 10^{14} \mathrm{~cm}^{-2}$ начинает эффективно работать механизм рекомбинации образующихся точечных дефектов. Возможно, этим и объясняется отсутствие после имплантации с дозой $5 \cdot 10^{15} \mathrm{~cm}^{-2}$ аморфизованного слоя, который, согласно TRIM-расчетам, должен был образоваться при достижении дозы $1.5 \cdot 10^{15} \mathrm{~cm}^{-2}$.

На рис. 2 также показан спектр исходного (до имплантации) образца, измеренный в каналирующем режиме (кривая 5). Он практически совпадает с каналированным спектром облученного с наибольшей дозой образца GaAs:N (кривая 4). Этот результат может быть обусловлен снятием напряжений, возникших при имплантации ионов азота с двумя

Письма в ЖТФ, 2018, том 44, вып. 13 
меньшими дозами, за счет образования протяженных дефектов во время имплантации с наибольшей дозой и возможным нагревом образца во время длительной имплантации. Однако необходимы дополнительные исследования для выяснения наблюдаемого явления.

Следует отметить, что предварительные исследования структурных свойств изученных в настоящей работе образцов методами рентгеновской дифрактометрии и просвечивающей электронной микроскопии подтверждают полученные результаты, свидетельствующие о совпадении каналированных RBS-спектров образцов, имплантированных с дозами $5 \cdot 10^{14}$ и $5 \cdot 10^{15} \mathrm{~cm}^{-2}$, а также исходного и облученного с наибольшей дозой образцов. Эти результаты выходят за рамки данной работы и будут представлены в отдельной публикации.

Таким образом, экспериментальные концентрационные профили атомов азота, измеренные методом SIMS, адекватно описываются зависимостями, рассчитанными с помощью программы TRIM. Анализ RBS-спектров образцов GaAs:N показал, что аморфизации имплантируемых слоев при дозах выше расчетного порога аморфизации не происходит. Профили концентрации обусловленных имплантацией точечных дефектов при дозах $5 \cdot 10^{14}$ и $5 \cdot 10^{15} \mathrm{~cm}^{-2}$ совпадают и имеют ряд особенностей. В приповерхностном слое глубиной меньше $200 \mathrm{~nm}$ обнаружена избыточная по сравнению с данными TRIM-расчетов их концентрация, которая уменьшается по мере увеличения глубины. Измеренные в каналирующем режиме RBS-спектры исходного и имплантированного с самой большой дозой $5 \cdot 10^{16} \mathrm{~cm}^{-2}$ образцов GaAs практически совпадают. Это означает, что после имплантации ионов азота с указанной дозой в образцах GaAs:N отсутствуют точечные дефекты, регистрируемые с помощью метода RBS. После проведения имплантации ионов азота необходим тщательный контроль присутствия на поверхности эпитаксиальных слоев GaAs избыточных атомов галлия и обогащенных галлием микротрубок.

Работа поддержана Российским научным фондом (проект № 17-19-01200). SIMS-измерения проводились на оборудовании ЦКП „Наука о материалах и диагностика перспективных технологий“ (ФТИ им. А.Ф. Иоффе РАН), поддержанного Министерством образования и науки РФ (уникальный идентификатор проекта RFMEFI62117X0018).

Авторы выражают благодарность В.Н. Пантелееву за предоставление образцов с эпитаксиальными слоями.

4 Письма в ЖТФ, 2018, том 44, вып. 13 


\section{Список литературы}

[1] Yu K.M., Walukiewicz W., Scarpulla M.A., Dubon O.D., Wu J., Jasinski J., Liliental-Weber Z., Beeman J.W., Pillai M.R., Aziz M.J. // J. Appl. Phys. 2003. V. 94. N 2. P. $1043-1049$.

[2] Мамутин В.В., Егоров А.Ю., Крыжсановская Н.В., Михрин В.С., Надточий А.М., Пирогов Е.В. // ФТП. 2008. Т. 42. В. 7. С. 823-830.

[3] Жуков А.Е., Семенова Е.С., Устинов М.B., Weber E.R. // ЖТФ. 2001. Т. 71. B. 10. C. $59-64$.

[4] Weyers M., Sato M., Ando H. // Jpn. J. Appl. Phys. 1992. V. 31. Pt 2. N 7A. P. L853-L855.

[5] Majlinger Z., Bozanic A., Petravic M., Kim K.-J., Kim B., Yang Y.-W. // Vacuum. 2010. V. 84. N 1. P. 41-44.

[6] Mikoushkin V.M., Bryzgalov V.V., Gordeev Yu.S., Nikonov S.Yu., Solonitsina A.P., Zhuravleva A.A., Brzhezinskaya M.M. // Phys. Status Solidi C. 2009. V. 6. N 12. P. 2655-2657.

[7] Gao K., Prucnal S., Skorupa W., Helm M., Zhou S. // J. Appl. Phys. 2013. V. 114. N 9. P. 093511.

[8] Gao K., Prucnal S., Skorupa W., Helm M., Shengqiang Zhou S. // Appl. Phys. Lett. 2014. V. 105. N 1. P. 012107.

[9] Mikoushkin V.M. // Nucl. Instr. Meth. Phys. Res. B. 2015. V. 354. N 1. P. 100 104.

[10] Ziegler J.F., Biersack J.P., Littmark U. The stopping and range of ions in solids. N.Y.: Pergamon Press, 1985. 321 p.

[11] Feldman L.C., Mayer J.W., Picraux S.T. // Material analysis by ion channeling. N.Y.: Academic Press, 1982. Ch. 5.

[12] Баранов Е.Е., Емельянов А.М., Лундин В.В., Петров В.Н., Сахаров В.И., Серенков И.Т., Соболев Н.А., Титков А.Н., Шек Е.И., Шмидт Н.М. // ЖТФ. 2006. T. 76. B. 12. C. 61-64.

[13] Соболев Н.А., Емельянов А.М., Лундин В.В., Сахаров В.И., Серенков И.Т., Усиков А.С., Емельянов А.М. // ФТП. 1999. Т. 33. В. 6. С. 674-676. 